\title{
The Use of Battlefield Acupuncture Prior to Botulinum Toxin A Administration: A 2-Patient Case Series
}

\author{
Xiangrong Shao, MD, FACP, ${ }^{1,2}$ Michael Corcoran, MD, FAAMA, and Michael O'Bryan, MD $^{4}$
}

\begin{abstract}
Background: Botulinum toxin type A injection is a common and safe procedure used for the treatment of overactive muscles through local injection. This toxin inhibits the release of acetylcholine in the neuromuscular junction. The benefits usually last only 3-6 months; thus, repeated injections are often required. The procedure, however, can be difficult if a patient's spasticity and pain prevents access to the muscles for injection or if a patient is anxious. Battlefield Acupuncture (BFA), a technique developed by Richard C. Niemtzow, MD, PhD, $\mathrm{MPH}$, in 2001, is a form of auricular acupuncture using a very specific sequence of gold Aiguille semipermanente needles inserted into the ear. BFA can be very effective for reducing pain quickly, with few potential side-effects. Cases: BFA was performed prior to Botulinum toxin A injections on 2 patients who had either pain limitations or anxiety limitations during prior Botulinum toxin A injections. Case 1 was a 70-year-old male veteran with painful, right upper-extremity spasticity with hand contractures. Case 2 was a 69 -year-old male veteran with spasticity who had anxiety related to his fear of needles.

Results: Application of BFA prior to Botulinum toxin A injections enabled the 2 patients who either had pain limitations or anxiety limitations to tolerate the toxin injections much better.

Conclusions: BFA is a safe and effective treatment option for rapid pain reduction, enabling Botulinum toxin A to be administered more easily to patients who have had pain or anxiety during prior injections.
\end{abstract}

Keywords: Battlefield Acupuncture, CVA, pain management, Botulinum toxin A injection

\section{INTRODUCTION}

\section{Background}

B OTULINUM TOXIN TYPE A INJECTION is a common and safe procedure used for the treatment of overactive muscles through local injection by inhibiting the release of acetylcholine in the neuromuscular junction. ${ }^{1,2}$ The benefits usually last only 3-6 months, and this requires reinjection of the toxin. The procedure, however, can be difficult if a patient's spasticity or pain prevent easy access to the muscles for injection, or if a patient has anxiety related to needles and is unable to remain still.

Acupuncture has been used for more than 5000 years, originating in China. This modality has been shown to have both local and distant effects within the body through the

\footnotetext{
${ }^{1}$ VA Maryland Health Care System, Geriatric and Extended Care Service, Loch Raven Medical Center, Baltimore, MD.

${ }^{2}$ University of Maryland, School of Medicine, Baltimore, MD.

${ }^{3}$ VA Maryland Care System, Physical Medicine and Rehabilitation Service, Baltimore, MD.

${ }^{4}$ Sinai Hospital, Baltimore, MD.
}

This material is the result of work supported with resources and the use offacilities at the VA Maryland Health Care System, Baltimore, $M D$. The contents do not represent the views of the U.S. Department of Veterans Affairs or the United States government. 
release of neurotransmitters and effects on the brain, as demonstrated on functional MRI. ${ }^{3}$ Battlefield Acupuncture (BFA) - an auricular acupuncture technique developed by Richard C. Niemtzow, MD, PhD, MPH, in 2001, while he was on active duty in the United States Air Force-is a form of auricular acupuncture using a very specific sequence of needles inserted into the ears; BFA has been shown to reduce pain in a few minutes with few potential side-effects. $^{4-6}$

\section{CASES}

\section{Case \#1}

A 70-year-old male veteran had a history of left middle cerebral artery strokes in 1991 and 1994, resulting in right upper-extremity spasticity with hand contractures. These contractures caused chronic recalcitrant pain. This patient was referred for local Botulinum toxin A injections. However, he was unable to have his upper extremity positioned for the injections due to his pain. After written informed consent was obtained from this patient, BFA was performed with gold Aiguille semipermanente needles being placed in the bilateral Cingulate Gyri and Thalami points on his ear, which reduced his spasm and decreased his pain level to facilitate the positioning needed for the for Botulinum toxin A injections. The patient reported that his pain went from a $6 / 10$ to a 4/10 after BFA. Botulinum toxin A injections were then performed, with 75 units in the extensor carpi radialis, 25 units in the flexor digitorum profundus (FDP) superficial, 25 units in the FDP deep, 50 units in the flexor digitorum superficialis, and 25 units in the flexor pollicis brevis. This patient tolerated each injection. The remaining 3 points of BFA were then performed bilaterally on him, and he reported his pain level as 1/10.

\section{Case \#2}

A 69-year-old male veteran with a history of a right pontine stroke in 1999, with likely two additional strokes since, had resulting right-sided spastic hemiplegia, leading to chronic right-sided posterior knee pain secondary to spasticity. The pain and spasticity had previously been welltreated with Botulinum toxin A; therefore, he was referred for repeat local toxin injections. During prior treatments, this patient had been very anxious while receiving the injections, leading to the concern that he would move during the repeat injections. While giving written informed consent, this patient remarked repeatedly about his fear of the injection needles. BFA was performed with gold ASP needles placed in the bilateral Cingulate Gyri and Thalami points. This patient tolerated the BFA with no anxiety or adverse events. He became mildly sedated and reported having less pain. He then tolerated the Botulinum toxin A injections, with 100 units in the biceps femoris in 2 locations, 100 units in the semimembranosis in 2 locations, and 100 units in the semitendinosis in 2 locations, without any movement.

\section{RESULTS}

Giving both of these patients BFA prior to Botulinum toxin A injections enabled the 2 patients tolerate the injections much better. In Case 1, the patient's pain was relieved enough for him to receive the toxin and, in Case 2, the patient's anxiety was ameliorated enough for him to also receive the toxin.

\section{DISCUSSION}

BFA is an emerging whole-body approach for the treatment of chronic pain and anxiety that are not adequately treated by conventional medicine. BFA's quick and easy application makes it appealing to trained practitioners to use prior to procedures that are painful or anxiety-provoking. A randomized controlled trial has shown that auricular acupuncture was no different than intranasal midazolam and was superior to no intervention for reducing dental anxiety. ${ }^{5}$ BFA has also been shown to reduce sore-throat pain for 24 hours and decrease the use of pain medication for up to 48 hours in a randomized controlled trial. ${ }^{6}$

\section{CONCLUSIONS}

BFA is a safe and effective treatment option for rapid pain reduction. Even frail geriatric patients can tolerate BFA with minimal-to-no side-effects.

In the 2 current cases, it was found that application of BFA prior to Botulinum toxin A injections enabled patients who either had pain limitations or anxiety limitations to tolerate the toxin injections with much more ease. While both the patients and practitioners were not blinded, the effects noted in these 2 cases were profound and worthy of further exploration of the use of BFA prior to Botulinum toxin A injection in selected patients.

\section{AUTHOR DISCLOSURE STATEMENT}

No competing financial interests exist.

\section{REFERENCES}

1. Naumann M, Jankovic J. Safety of botulinum toxin type A: A systematic review and meta-analysis. Curr Med Res Opin. 2004;20(7):981-990. 
2. Munchau A, Bhatia KP. Uses of botulinum toxin injection in medicine today. BMJ. 2000;320(7228):161-165.

3. Hui KK, Liu J, Makris N, et al. Acupuncture modulates the limbic system and subcortical gray structures of the human brain: Evidence from fMRI studies in normal subjects. Hum Brain Mapp. 2000;9(1):13-25.

4. Niemtzow R, Belard J-L, Nogier R. Battlefield Acupuncture in the U.S. military: A pain-reduction model for NATO. Med Acupunct. 2015;27(5):344-348.

5. Karst M, Winterhalter M, Munte S, et al. Auricular acupuncture for dental anxiety: A randomized controlled trial. Anesth Analg. 2007;104(2):295-300
6. Moss D, Crawford P. Ear acupuncture for acute sore throat: A randomized controlled trial. J Am Board Fam Med. 2015; 28(6):697-705.

Address correspondence to: Xiangrong Shao, MD, FACP VA Maryland Health Care System Geriatric and Extended Care Service Loch Raven Medical Center 3900 Loch Raven Boulevard Baltimore, MD 21218

E-mail: Xiangrong.shao@va.gov 